\title{
Genetic factors: influence on the development of thyroid diseases and rheumatic diseases
}

\begin{abstract}
The article discusses the presence of common genes in RA and thyroid disease.

Methods: Published studies that enrolled adult patients with rheumatoid arthritis (RA) and autoimmune thyroid pathology were identified by searches of Medline and Embase, and by manual searches of medical journals. Meta-analyses were performed to assess the association of SE presence, dose, and genotype with rheumatoid arthritis and autoimmune thyroid pathology.
\end{abstract}

Results: A total of studies 133 patients were included in the analysis. RA patients have increase in the frequency of the Gene DR B1 $(\mathrm{p}<0.05, \mathrm{OR}=3,2,95 \% \mathrm{CI}=1,4-6,8)$, the gene $\mathrm{B} 8(\mathrm{p}<0.05, \mathrm{RR}=2,6 ; 95 \% \mathrm{CI}=1,8-4,3)$. In patients with autoimmune thyroid disease, most frequently found genes HLA-B8 (94\% patients) HLA-DR3 $(67 \%)$ and HLA-DR5 (72\%).

Conclusion: According to the obtained results it is concluded that the presence of the HLA - B8 gene can cause of RA in patients with autoimmune thyroid pathology.

\author{
Special Issue - 2018
}

\author{
Paramonova OV \\ Volgograd State medical University, Russia
}

Correspondence: Paramonova OV,Volgograd State medical University, Russia, Email stella243@mail.ru

Received: May 31, 2017 | Published: November 15, 2018

\section{Introduction}

Autoimmune diseases are diseases associated with dysfunction of the human immune system, which perceives its own tissues as foreign and damaging it. Such diseases are called systemic, because, as a rule, affects the whole system or even the entire body. The incidence of autoimmune rheumatic diseases and their prevalence is sufficiently large in the world. The most common among the adult population is rheumatoid arthritis. ${ }^{1}$ Rheumatoid arthritis (RA) is an autoimmune rheumatic disease of unknown etiology, characterized by chronic and erosive arthritis and systemic lesions of internal organs. ${ }^{1}$ The role of genetic factors in the development of the disease has been is confirmed by various studies on immune genetics. ${ }^{2-4}$ It is estimated that about $40 \%$ of the total genetic component of RA refers to the major histo compatibility complex the HLA system. ${ }^{5}$ A detailed analysis of the associations of RA with the HLA system suggests that HLA alleles are associated not only with sensitivity but also with the protection (resistant) from the development of the disease. ${ }^{6-8}$ RA corresponds to the multi factorial polygenic model of inheritance with typical consequences in the family, a genetic marker of disease predisposition and variability of clinical manifestations, depending on gender and age. Genetic factors involved in the development of RA, along with such factors as viral or bacterial infection, joint injuries, hypothermia, sun exposure, medications, etc.). ${ }^{9}$ Pathogenesis of rheumatoid arthritis is a violation of the presentation of antigens. A dominant role in the selection and presentation of antigens in cooperation of immune cells with HLA genes and antigens, encoded. According to the literature, in European population Association established rheumatoid arthritis adults with the genes of the Gene locus DRB $1 * 01, * 03, * 04{ }^{2,3}$ The aim of our work was to identify similarities in the pathogenesis of RA and autoimmune thyroid disease to determine the cause of the very frequent thyroid lesions in RA.

\section{Material and methods}

In the study, we surveyed 133 people, 100 of them diagnosed with RA, 33 with RA and autoimmune thyroid disease. Typing genes HLA class I and class II loci DRB1, DQA1, DQB1 was performed by PCR using sets of reagents of firm "DNA Technology" and the sequence of a set of primer. ${ }^{8}$ Statistical processing included calculation of the frequency of occurrence of the Gene (PX) and antigens $(\mathrm{C} \%)$ is calculated in accordance with the law of hardy-Weinberg equilibrium, Pearson's $\mathrm{x}^{2}$ with Yates's correction for continuity and the criterion odds ratio.

\section{Results and discussion}

\section{The group of patients with RA}

Among the total number of examined patients with RA were 83 women and $17 \mathrm{men}$. All patients were divided into groups according to the classification of rheumatoid arthritis. ${ }^{1}$ The age of the patients was from 20 to 75 years, mean age was $52.3 \pm 11.4$ years. Disease onset age from 16 to 70 years, average age of onset of the disease amounted to $42.4 \pm 14.8$ years.

\section{The group of patients with pathology of the thyroid gland}

Under our supervision there were 33 patients with pathology of autoimmune thyroid pathology, 5 of them men and 28 women, which confirms the data about greater prevalence of thyroid disease in women than in men $[9,6]$. The average age of the patients was 52 , $48 \pm 14.03$ years. The average duration of the presence of clinically expressed thyroid disease $-3,82 \pm 3.21$ years. The onset is on average $48.6 \pm 13.6$ years. In the presence of patients with thyroid disease as follows: chronic autoimmune Thyroiditis, euthyroid-31\%, primary hypothyroidism and $27 \%$, diffuse toxic goiter-30\%, mixed toxic goiter $-12 \%$. In our study when comparing groups of patients with RA revealed statistically significant increase in the frequency of the Gene DR B1 $(\mathrm{p}<0.05, \mathrm{OR}=3,2,95 \% \mathrm{CI}=1,4-6,8)$, the gene $\mathrm{B} 8(\mathrm{p}<0.05$, $\mathrm{RR}=2,6 ; 95 \% \mathrm{CI}=1,8-4,3)$. But HLA-DR5, HLA- DR2, HLA-DR3 and HLA-DR7 was found rarely, only in $8 \%$ of patients. In patients with autoimmune thyroid disease, most frequently found genes HLA-B8 (94\% patients) HLA-DR3 (67\%) and HLA-DR5 (72\%). 
Table I The frequency of detection of the studied genes

\begin{tabular}{lllll}
\hline Gene/\% positive & DR B I & B8 & DR3 & DR5 \\
\hline RA & 75 & 92 & 4 & 8 \\
RA+ autoimmune thyroid pathology & 34 & 94 & 67 & 72 \\
\hline
\end{tabular}

It is assumed that the gene HLA-DR5 may be involved in the mechanism of formation of goiter, while HLA-DR5 is the acceptor of T-suppressors and stimulates them to release of prostaglandins, and HLA-ДR3 calls a helper function of $\mathrm{T}$ lymphocytes. Hypertrophic form of autoimmune thyroidite in combination with genes HLA-DR5, while the atrophic form, with the genes HLA-DR3 and HLA-B8. Mechanism of Association of HLA genes is one of the variants of the Gene drb1 genotype dqa1 with RA was proposed in $1987 \mathrm{R}$. Gregersen. According to his theory, the clinical phenotype manifested by individual patients with RA, such as the presence of extraarticular manifestations, demonstrates substantial variability that may reflect genetic differences. The most extensively studied genetic marker in RA is HLA-DRB1 and, more specifically, those alleles with a conserved amino acid sequence in the molecule's third hypervariable region, termed the shared epitope (SE). Among individual studies, the most striking relationship of the SE to RA outcome was described in an analysis of the association with extraarticular manifestations in a highly selected group of RA patients. ${ }^{10}$ But still have not identified this peptide, which was only discovered T-helper and went to the same type of development of an immune response to specific target organs. However, some modified versions of the peptides closer to the desired model, making this explanation likely. However, the same sequence is also present in DRP 1 the contour of the HLA-a Gene variant Gene variant drb $1 * 03$, but its role in the pathogenesis of RA in our study is uncertain, which may be due to ethnic characteristics of the population.

Thus, it is clear that both diseases are associated with HLA-B8. The received materials allow assuming that certain genes and alleles of class II HLA class involved in the pathological process of autoimmune rheumatic diseases, but the way you implement it is different. As RA is a Multisystem autoimmune disease that can affect any target organ, some authors put forward the hypothesis that the mixed pathology of the auto stereogram can be the result of a group of antithyroid produced in Armenia. It is possible that the auto stereogram pathology is a consequence of the immune damage to the thyroid gland in rheumatoid arthritis, accompanied by the deposition of immune complexes and activation of the complement system.

\section{Conclusion}

Thus, the identified carriers of the predisposing gene (HLA-B8) is not an indication of a mandatory development of RA in the presence of auto threading disease and/or thyroid destruction in RA, but when exposed to certain factors may act as one of the causes of development of multi-organ autoimmune process. We can assume that a genetic predisposition to pathological autoimmune processes determines the ability of various cross-syndromes and combinations of systemic and organ-specific autoimmune diseases in one patient, which is a characteristic feature of this disease. In conclusion, it should be noted that the problem of thyroid disease in rheumatoid arthritis relevant. Controversial and sometimes contradictory literature data concerning the characteristics of the relationship of the thyroid gland and rheumatic diseases, suggest the need for further research in this area.

\section{Acknowledgements}

None.

\section{Conflict of interest}

The author declares no conflict of interest.

\section{References}

1. Nasonov EL. Clinical guidelines Rheumatology GEOTAR. USA: Springer; 2010. $752 \mathrm{p}$.

2. Nikulin S Yu, Chernov AA, Bolshakova TY, et al. Genes of predisposition to rheumatoid arthritis. Siberian medical. 2014;3:11-18.

3. Nikulina S Yu, Chernov AA, Bolshakova TY, et al. Genetic predictors of rheumatoid arthritis. RMJ. 2014;27:1986.

4. TuressonC, Matterson EL. Genetics of rheumatoid arthritis. Mayo Clin Proc. 2006;81(1):94-101.

5. Zaretskaya Yu, Lednev Yu. HLA 50 years. Tver Triad. 2008:152.

6. Paramonova OV. Clinical diagnostic value of determination of antibodies to thyroid hormones in patients with rheumatoid arthritis in combination with the defeat of the thyroid gland using magnetic immunosorbent. International Journal of endocrinology. 2010;5 (29):96-103.

7. Muthanaa M, Hawtreea S, Wilshawa A. C5orf30 is a negative regulator of tissue damage in rheumatoid arthritis. Proc Natl Acad Sci US A. 2015;112(37):11618-11623.

8. Chernecky CC, Berger BJ. Laboratory tests and diagnostic procedures. USA: Saunder Elsevier; 2008.

9. Paramonova OV, Rusanova OA, Gontar IP. The relationship of organspecific autoimmune thyroid disease non-autoimmune rheumatic diseases. Clinical and experimental Thyroidology. 2012;8(1):46-50.

10. Gorman J, David Vaudey E, et al. Particular HLA-DRB1 shared epitope genotypes are strongly associated with rheumatoid vacuities. Arthritis and rheumatism. 2004;50(11):3476-3484. 